\title{
Tachymenis peruviana Wiegmann, 1834 (Serpentes, Dipsadidae) in Argentina: geographic distribution and a new province record
}

\author{
Gabriela A. Gallardo ${ }^{1}$, Santiago J. Nenda ${ }^{2}$, Gustavo J. Scrocchi Manfrini ${ }^{3}$ \\ 1 Dpto. de Cs. Básicas y Tecnológicas. Instituto de Ambientes de Montañas y Regiones Áridas. Universidad Nacional de Chilecito. Ruta Los \\ Peregrinos s $/ \mathrm{n}^{\circ}-\mathrm{CP}$ (5360). Chilecito, La Rioja, Argentina. 2 División Herpetología, Museo Argentino de Ciencias Naturales "Bernardino \\ Rivadavia", Consejo Nacional de Investigaciones Científicas y Técnicas, Avenida Ángel Gallardo 470, C1405DJR Buenos Aires, Argentina. 3 \\ UEL-CONICET, Fundación Miguel Lillo. Miguel Lillo 251- (4000). San Miguel de Tucumán. Tucumán. Argentina. \\ Corresponding author: Gustavo J. Scrocchi, gustavo.scrocchi@gmail.com
}

\begin{abstract}
Tachymenis peruviana Wiegmann, 1834 (Serpentes, Dipsadidae) is known from Peru, Bolivia, northern Chile and northwestern Argentina. In northwestern Argentina, the species is distributed from Jujuy to Catamarca province. In this study, we present the accurate distribution of the species in Argentina and the first records from La Rioja province, at 2000 and $3000 \mathrm{~m}$ a.s.l. in the Famatina region, extending the known distribution to the southwest.
\end{abstract}

Key words

Highlands, snakes, Tachymenini.

Academic editor: Edward A. Myers | Received 13 September 2018 | Accepted 29 November 2018 | Published 4 January 2019

Citation: Gallardo GA, Nenda SJ, Scrocchi Manfrini GJ (2019) Tachymenis peruviana Wiegmann, 1834 (Serpentes, Dipsadidae) in Argentina: geographic distribution and a new province record. Check List 15 (1): 7-13. https://doi.org/10.15560/15.1.7

\section{Introduction}

Species of the genus Tachymenis are small to moderate-sized snakes, distributed from Peru to southern Argentina and Chile. The genus is represented in Argentina by 2 taxa: Tachymenis chilensis chilensis, known to inhabit southern Chile and the Argentine provinces of Neuquén, Río Negro, and Chubut (Scrocchi et al. 2010, Giraudo et al. 2012a, b, Nenda et al. 2017), and Tachymenis peruviana, which was known until now in Peru, Bolivia, northern Chile, and the northwestern Argentine provinces of Jujuy, Salta, Tucumán and Catamarca (Cei 1993, Giraudo and Scrocchi 2002, Scrocchi et al. 2006, Giraudo et al. 2012a).

In this wide range of distribution, $T$. peruviana dwells from 1800 to $4400 \mathrm{~m}$ a.s.l. in areas with shrubs and grasslands from the biogeographic provinces of Yungas, Prepuna, Puna, and Altoandina (Aparicio 1994, Cabrera and Willink 1980, Giraudo and Scrocchi 2002). Tachymenis peruviana is viviparous, diurnal species, frequently found near rivers, lagoons, small streams, where it feeds on anurans (Cei 1993, Williams and Scrocchi 1994, Scrocchi et al. 2006, Szumik et al. 2016, Demangel 2016, Cabrera et al. 2017) or in sandy or rocky areas, where it feeds on lizards. This opistoglyph snake has strong toxicity and fatalities by bites were reported for its relative T. chilensis (Scrocchi et al. 2006).

The species is a generalist in habitat use and is commonly found, evaluated as "Not threatened" in Argentina (Giraudo et al 2012b), and Least Concern by the IUCN (2018). The detailed distribution of this species is not known in Argentina. 


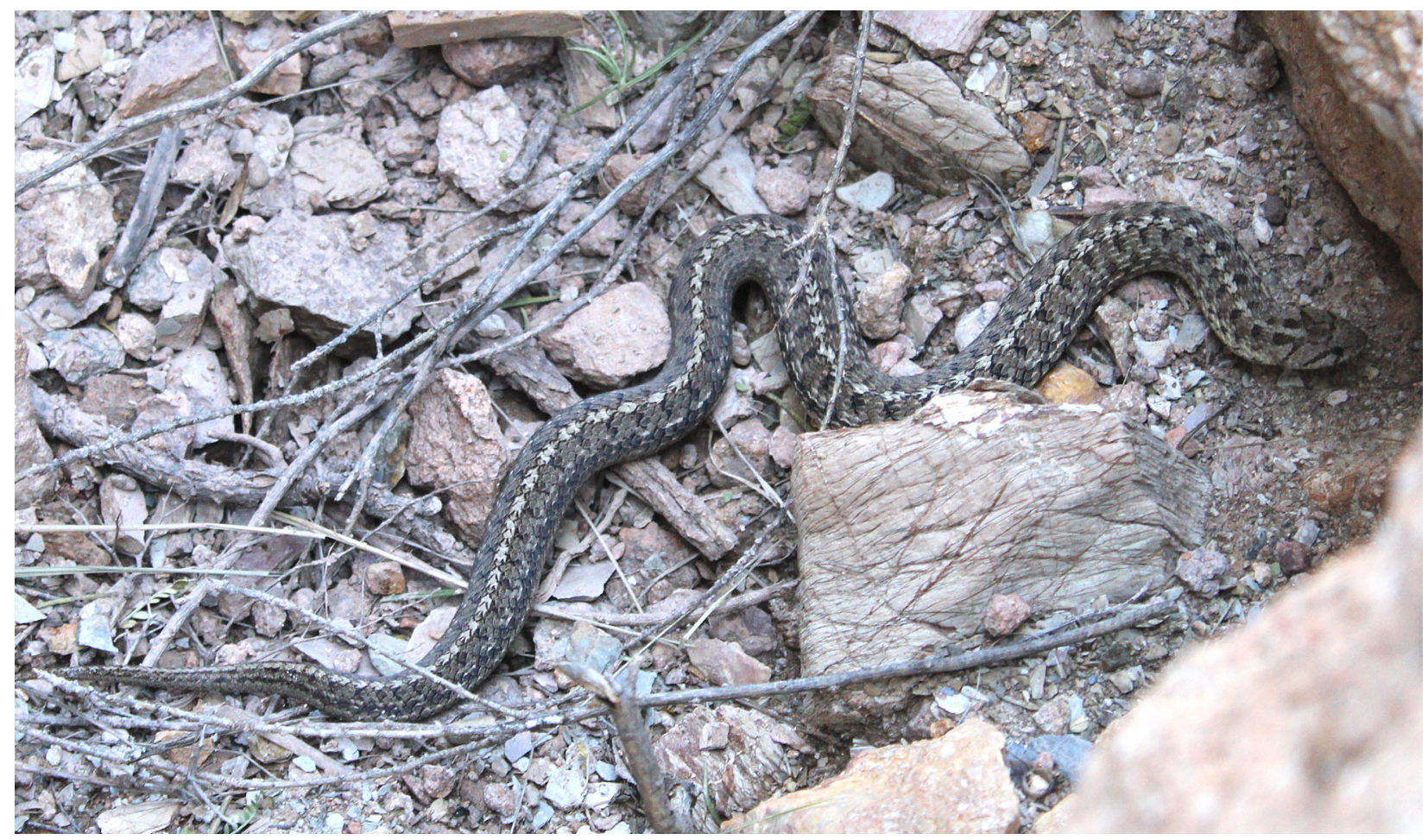

Figure 1. Tachymenis peruviana. Specimen from Cueva de Logroño. La Rioja. Argentina.

\section{Methods}

We revised 112 specimens of the species, which belong to the following Argentine museums:

CENAI. Centro Nacional de Investigaciones Iológicas. Housed at División Herpetología, Museo Argentino de Ciencias Naturales "Bernardino Rivadavia", Buenos Aires, Argentina.

FML. Herpetological Collection of Fundación Miguel Lillo, San Miguel de Tucumán, Argentina.

IBIGEO-R. Instituto de Bio y Geociencias del NOA. CONICET-Universidad Nacional de Salta, Salta, Argentina.

MACN. Herpetological Collection of Museo Argentino de Ciencias Naturales "Bernardino Rivadavia", Buenos Aires, Argentina.

MCN-UNSa. Museo de Ciencias Naturales, Universidad Nacional de Salta. Salta. Argentina.

The specimens were identified using the characters mentioned by Cei (1993) and Scrocchi et al. (2006). We use the decimal geographic coordinates and altitudes of the collections databases, rounded to 4 decimal places.

\section{Results}

The revision of the specimens allowed us to present the distribution of the species in the country and also the first records from La Rioja province (Other material examined and Figures 2-4).

New records. ARGENTINA. La Rioja: Departamento Chilecito: El Parrón, 3rd Station of the cableway from Chilecito to La Mexicana mine, $\left(-29.0804^{\circ},-67.6425^{\circ}\right.$,
2000 m a.s.1., G. A. Gallardo, 01/28/2015, 1 ex. FML 30163 ); found active at $18: 45 \mathrm{~h}$. A second specimen (Fig. 1, no voucher specimen) was seen in Departamento

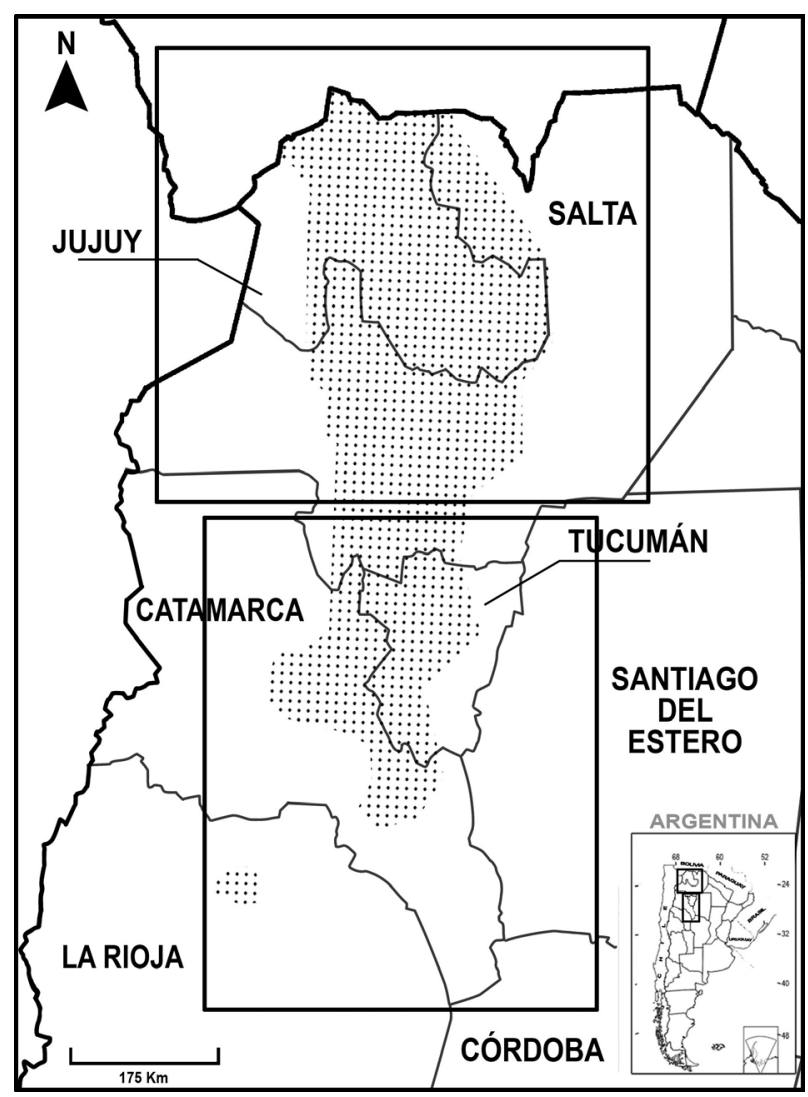

Figure 2. General distribution of Tachymenis peruviana in Argentina. The northern area is shown in Figure 3 and the southern in Figure 4. The disjunct area represents new records. 


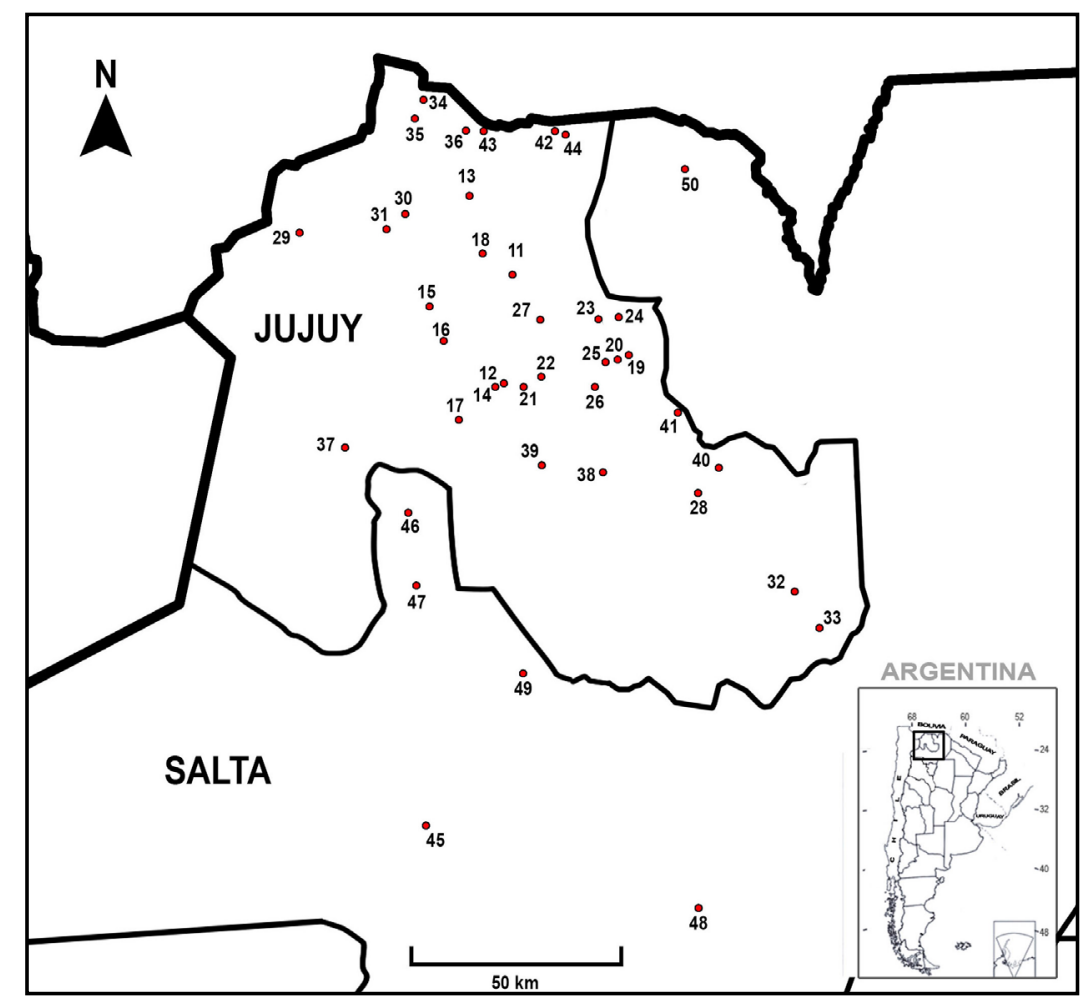

Figure 3. Northern localities in the distribution of Tachymenis peruviana from Argentina. The numbers refer to material examined.

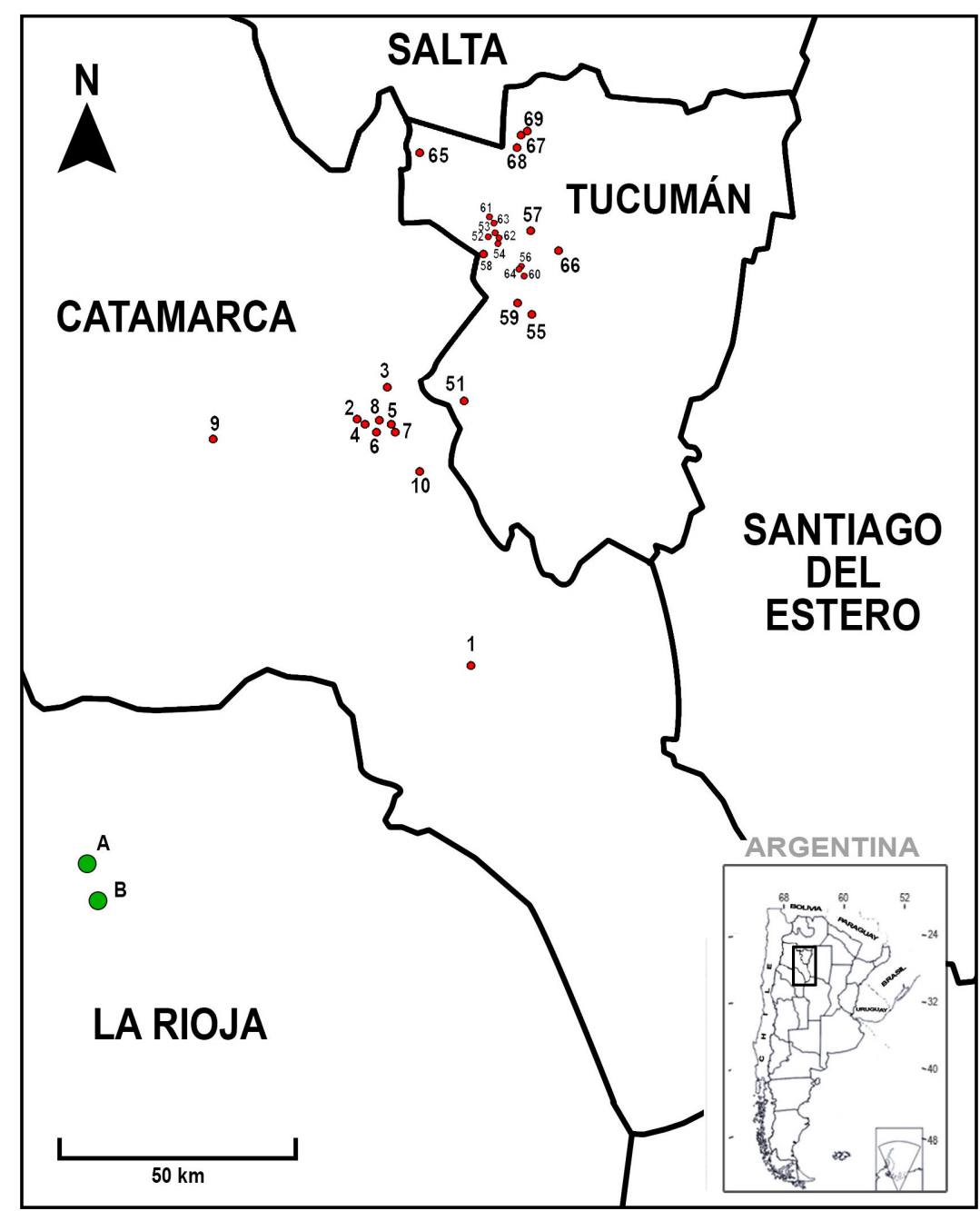

Figure 4. Southern localities in the distribution of Tachymenis peruviana from Argentina. The numbers refer to material examined. Green dots represent the new locality records from La Rioja province: A. Cueva de Logroño. B. El Parrón (FML 30163). 
Famatina: Cueva de Logroño $\left(-28.9350^{\circ},-67.6842^{\circ}\right.$, $3000 \mathrm{~m}$ a.s.l. photographed by E. Luna Toledo, 04/14/2011), near an ocher mine. Both localities are on the Sistema de Famatina, an extra-andean geologic system located from $27^{\circ}$ to $31^{\circ} \mathrm{S}$ in northwestern Argentina, with elevations that reaches $6000 \mathrm{~m}$ a.s.l. (Ramos 1999).

Other material examined. Figure 2 shows the general distribution of Tachymenis peruviana in Argentina. The numbers that precede each locality serve as reference for Figures 3 and 4.

ARGENTINA. Catamarca: Tranquitas (not on the map, without collector information, 1950, MACN10283). Departamento Ambato: 1. Cerro El Manchao (-28.2864 $-65.9844^{\circ}, 2300 \mathrm{~m}$ a.s.1., R.F. Laurent, 10/22-29/1970, 1 ex. FML00621, A. L. Ocampos, 01/19/1971, 1 ex., CENAI2955); Río Los Cajones, Cerro El Manchao $\left(-28.2810^{\circ},-66.0202^{\circ}, 3100 \mathrm{~m}\right.$ a.s.1. S. Halloy, 10/23/ 1979, 1 ex., FML01155); and La Cumbrecita, Cerro El Manchao $\left(-28.2865^{\circ},-66.0122^{\circ}, 2800 \mathrm{~m}\right.$ a.s.l. O. Pagaburo, O. Budin, 10/25/1979, 1 ex. FML01156). Departamento Andalgalá: 2. Capillitas $\left(-27.3463^{\circ},-66.3902^{\circ}\right.$, 3500 m a.s.l. R.F. Laurent. 02/21/1968, 1 ex. FML00571; E. Terán, O. Pagaburo, 03/18/1982, 1 ex. each FML0134401345; G. Scrocchi, G. Gallardo, 02/14/2006, 1 ex. FML16986). 3. El Arenal, $19 \mathrm{~km}$ al NE de Capillitas $\left(-27.2305^{\circ},-66.2738^{\circ}\right.$, R.F. Laurent, 02/21-23/1968, 1 ex. FML00586; R.F. Laurent, 01/21-23/1968, 1 ex. FML00624). 4. Quebrada La Toma, Capillitas (-27.3538 , $-66.3740^{\circ}, 3200 \mathrm{~m}$ a.s.l. E. Terán, O. Pagaburo 03/23/1982, 1 ex. FML01343). 5. Puesto Río Candado $\left(-27.3964^{\circ},-66.2556^{\circ}\right.$, G. Scrocchi, 05/20/1997, 1ex. FML06600). 6. Cerca de El Globo (-27.3541 ${ }^{\circ}$, $-66.3234^{\circ}, 4000 \mathrm{~m}$ a.s.1., G. Scrocchi, 03/17/1998, 1ex. FML07135). 7. Quebrada Melcho, Campamento Agua Rica $\left(-27.3833^{\circ},-66.2666^{\circ}\right.$, J. Brancato, C. Boero, 03/19/1998, 1 ex. FML07139). 8. Filo de Loma Baya, Área de Cumbre $\left(-27.3822^{\circ},-66.3355^{\circ}, 3100 \mathrm{~m}\right.$ a.s.1., J. Brancato, 03/20/1998, 1 ex. FML07142). 9. La Banderita $\left(-27.3469^{\circ},-65.9775^{\circ}\right.$, R.F. Laurent, 1968, 1 ex. each, FML00582, and FML 00587). Departamento Belén: 10. Las Pampitas, entre Farallón Negro y Bajo La Alumbrera, Distrito Hualfín $\left(-27.5332^{\circ},-66.1502^{\circ}\right.$, E. Terán, O. Pagaburo, 03/12/1982, 1 ex. FML01346). Departamento Tinogasta: Campamento El Potrerito, La Ciénaga, Medanitos (not in the map, O. Pagaburo, 03/12/1987, 1 ex. FML01687). Jujuy: Departamento Cochinoca: 11. Abra Pampa $\left(-22.7205^{\circ},-65.6970^{\circ}\right.$, E. Maury, 01/12/1966, 1 ex., MACN25158). 12. Estafeta de Abra Laite ( $-23.2001^{\circ}$, $-65.7833^{\circ}$, without date or collector information, 1 ex., CENAI2635; F. Contino, 01/071969, 1 ex., CENAI2636 paratypes of Tachymenis peruviana yutoensis Miranda and Couturier a junior synonym of T. peruviana). 13. Puesto Miguel Garay, Camino a Cerro Chorrión, al E de Laguna Pozuelos, $\left(-22.3821^{\circ},-65.8685^{\circ}, 3850 \mathrm{~m}\right.$ a.s.1., E. Lavilla, 12/14/1979, 1 ex. FML01191). 14. Agua de Castilla, vertiente $\mathrm{W}$ de Sierra Aguilar $\left(-23.2062^{\circ}\right.$, -65.7977 , E. Lavilla, 11/26/1981, 1 ex. FML01313). 15.
Rachaite $\left(-22.8370^{\circ},-66.0742^{\circ}\right.$, J. Baldo, Y. Arzamendia, 04/10/2000, 1 ex. FML17643). 16. Casabindo $\left(-22.9852^{\circ},-66.0325^{\circ}\right.$, without collector information 04/28/1996, 1 ex. FML17646). 17. Alfarcito (-23.3196º, -65.9740 ${ }^{\circ}$, J. Baldo, 04/04/2009, 1 ex. FML23553). 18. Ruta Provincial 7; 4 km al SE de Tres Arbolitos, próximo a Laguna Pozuelos $\left(-22.6226^{\circ},-65.8233^{\circ}, 3650 \mathrm{~m}\right.$ a.s.1., C. Szumik, D. Ríos, 05/06/2017, 1 ex. FML29948). Departamento Humahuaca: 19. Entre Aparzo y Palca de Aparzo (-23.0909,$-65.1876^{\circ}$, E. Lavilla, 11/24/1979, 1 ex. FML01169). 20. Entre Aparzo y Humahuaca $\left(-23.1114^{\circ},-65.2336^{\circ} 4300 \mathrm{~m}\right.$ a.s.1., G. Scrocchi et al., 03/03/2007, 1 ex. FML18536). 21. El Aguilar, Mina Aguilar $\left(-23.2091^{\circ},-65.6682^{\circ}, 4000 \mathrm{~m}\right.$ a.s.1., E. Guanuco, 12/18/1981, 1 ex. FML01315). 22. Quebrada La Toma, El Aguilar, camino a la Repetidora $\left(-23.1613^{\circ}\right.$, $-65.5904^{\circ}, 4000 \mathrm{~m}$ a.s.1., O. Pagaburo, 01/11/1988, 1 ex. FML01789). 23. Chaupi Rodeo, ruta a Iruya (-22.9310 ${ }^{\circ}$, $-65.3111^{\circ}$, $3450 \mathrm{~m}$ a.s.1., O. Pagaburo, 01/20/1988, 1 ex. FML01790). 24. Quebrada Tonocote, subiendo por Chaupi Rodeo a Laguna Leandro, Chorcán $\left(-22.9327^{\circ}\right.$, -65.2256 ${ }^{\circ}, 4100-4400 \mathrm{~m}$ a.s.1., O. Pagaburo, 01/22/1988, 2 ex. FML01791). 25. Coctaca $\left(-23.1227^{\circ},-65.2867^{\circ}\right.$, Casanova, Gallo, without date information, 1 ex. each, MACN 6157-6161). 26. Humahuaca (-23.2122 $-65.3512^{\circ}$, De Carles, without date information, 1 ex., MACN3004). 27.Tres Cruces $\left(-22.9203^{\circ},-65.5814^{\circ}\right.$, Cia. Minera, 04/16/1971, CENAI3018 paratype of T. $p$. yutoensis). Departamento Ledesma: 28. Abra Colorada, Parque Nacional Calilegua $\left(-23.6880^{\circ},-64.9077^{\circ}, \mathrm{M}\right.$. Vaira, 02/1999, 1 ex. FML09075). Departamento Rinconada: 29. Vilama, Cuesta del Farallón. Camino de cornisa entre el pueblo Lagunillas del Farallón y Vilama $\left(-22.4945^{\circ},-66.643^{\circ}, 4400 \mathrm{~m}\right.$ a.s.1., M. Akmentins, J. Urquizo, 03/09/2016, 1 ex. IBIGEO-R5453). 30. Rinconada $\left(-22.4415^{\circ},-66.1645^{\circ}, 3800 \mathrm{~m}\right.$ a.s.l., O. Pagaburo et al., 01/25/1984, 2 ex. FML01510). 31. Fundiciones, 40 $\mathrm{km}$ NE de Mina Pirquitas $\left(-22.5011^{\circ},-66.2501^{\circ}\right.$, V. Abdala et al., 01/15/1989, 3 ex. FML01969). Departamento Santa Bárbara: 32. Sierra de Santa Bárbara $\left(-24.1167^{\circ},-64.4833^{\circ}\right.$, R.F. Laurent, 01/1966, 2 ex. FML01018). 33. Cerro Centinela $\left(-24.2784^{\circ},-64.3780^{\circ}\right.$, A. Vilte, 12/18/2011, 1 ex. FML25078). Departamento Santa Catalina: 34. Ladera NW Cerro Santa Catalina $\left(-21.9666^{\circ},-66.0500^{\circ}, 3600 \mathrm{~m}\right.$ a.s.1., O. Pagaburo et al. 01/15/1984, 1 ex. FML01509); 35. 12 km de Santa Catalina, camino a El Angosto $\left(-22.0429^{\circ},-66.0862^{\circ}, 3900\right.$ $\mathrm{m}$ a.s.l., O. Pagaburo et al., 01/15/1984, 1 ex. FML 01511). 36. Cieneguillas $\left(-22.1010^{\circ},-65.8613^{\circ}, \mathrm{J}\right.$. Baldo, 02/02/2004, 1 ex. FML17648). Departamento Susques: 37. Susques $\left(-23.4109^{\circ},-66.5022^{\circ}\right.$, E. Lavilla, 03/22/1980, 1 ex. FML01190). Departamento Tilcara: 38. Paraje Casa Colorada, entre Tilcara y San Francisco $\left(-23.5778^{\circ},-65.3329^{\circ}\right.$, F.G. Burgos, 04/24/2012, 1 ex. FML25071). Departamento Tumbaya: 39. Arroyo y Vega El Morado, Sierra Alta $\left(-23.5387^{\circ},-65.6151^{\circ}\right.$, J. Baldo, 02/08/2001, 1 ex. FML17644). Departamento Valle Grande: 40. Cerro Calilegua $\left(-23.5812^{\circ},-64.7998^{\circ}\right.$, 
J.M. Gómez, without date information, 2 ex. FML00620). 41. Abra del Valle, Santa Ana $\left(-23.3540^{\circ},-64.9767^{\circ}\right.$, J. Baldo, Y. Arzamendia, 04/28/2001, 1 ex. FML17645). Departamento Yavi: 42. $20 \mathrm{~km} \mathrm{~W}$ de La Quiaca, por ruta Provincial $5\left(-22.1461^{\circ},-65.4212^{\circ}, Z\right.$ Z Tomsic, 11/27/1974, 1 ex. FML00793). 43. Toqueros (-22.1177, $-65.7811^{\circ}$, D. Bald. Entre San Bernardo de las Zorras y El Rosal, Ruta Provincial 127, 21.3 km NE de Ruta Nacional 51 (-24.4025 -65.7768, 3300 m a.s.1., T. Hibbard et al., 04/07/2017, 1 ej. IBIGEO-R5717-5718). o, 06/05/2002, 1 ex. FML17647). 44. Yavi $\left(-22.1334^{\circ}\right.$, $-65.4611^{\circ}$, D. Baldo et al., 02/14/2005, 1 ex. FML17649). Salta: Departamento Cachi: 45. Quebrada Las Pailas, Cachi Adentro $\left(-25.0148^{\circ},-66.2573^{\circ}, 3300-3500 \mathrm{~m}\right.$ a.s.l., E. Pereyra, O. Pagaburo, 01/28/1985, 2 ex. FML01552). Departamento La Poma: 46. Cobres, $67 \mathrm{~km}$ $\mathrm{N}$ de San Antonio de Los Cobres $\left(-23.6891^{\circ},-66.2372^{\circ}\right.$, G. Scrocchi, 05/04/1977, 1 ex. FML00972). Departamento Los Andes: 47. $25 \mathrm{~km} \mathrm{~N}$ de San Antonio de los Cobres por ruta nacional $40(-24.0143-66.2378,3500 \mathrm{~m}$ a.s.l., L. Díaz Fernández et al., 12/20/2015, 1 ex. IBIGEO-R 5462). Departamento Metán: 48. Sierra de Metán, orillas del Río Vallecito $\left(-25.4845^{\circ},-65.0334^{\circ}\right.$, E. Terán, O. Pagaburo, 10/16/1977, 1 ex. FML00984). Departamento Rosario de Lerma: 49. Entre San Bernardo de las Zorras y El Rosal, Ruta Provincial 127, $21.3 \mathrm{~km}$ NE de Ruta Nacional 51 (-24.4025 -65.7768, 3300 m a.s.l., T. Hibbard et al., 04/07/2017, 1 ej. IBIGEOR5717-5718). Departamento Santa Victoria: 50. Cercanías de Cañani $\left(-22.3166^{\circ},-64.8833^{\circ}\right.$, A. Brunetti, F. Burgos, 02/02/2008, 1 ex. MACN51585). Tucumán: Departamento Chicligasta: 51. Entre Laguna del Tesoro y Cochuna $\left(-27.2900^{\circ},-65.9541^{\circ}, 1800 \mathrm{~m}\right.$ a.s.l., S. Halloy, 07/13/1974, 1 ex. FML00767). Departamento Tafí del Valle: 52. Cerro Muñoz (-26.7217,$-65.8080^{\circ}$, C.C. Olrog, 01/21/1954, 1 ex. FML00327). 53. El Molle, Sierra del Aconquija $\left(-26.7008^{\circ},-65.8012^{\circ}, 2900 \mathrm{~m}\right.$ a.s.1., R. Cei, 05/27/1967, 1 ex. FML00556). 54. El Infiernillo, camino a El Negrito $\left(-26.7366^{\circ},-65.7851^{\circ}\right.$, 3500 m a.s.1., C. Halloy, 12/1970, 1 ex. FML00607, A. Marcus et al., 01/31/1980, 1 ex. FML01171). 55. Puesto El Sunchal, km 41 de ruta a Tafí del Valle $\left(-26.9956^{\circ}\right.$, -65.6629ㅇ, J. M. Gómez, 10/28/1971, 1 ex. FML00644). 56. $\mathrm{Km} 68.5$ de la ruta provincial $307\left(-26.8126^{\circ}\right.$, $-65.7194^{\circ}$, R.F. Laurent, 04/12/1974, 1 ex. FML00750). 57. Ciénaga Grande, Cumbres Calchaquíes (-26.6834 ${ }^{\circ}$, -65.6504 ${ }^{\circ}$, S. Halloy, 12/29/1979, 1 ex. FML00960). 58 Ciénaga de los Caminos, Cerro Muñoz $\left(-26.7689^{\circ}\right.$, $-65.8494^{\circ}, 3850 \mathrm{~m}$ a.s.1., A. Marcus et al., 01/31/1980, 1 ex. FML01171). 59. El Mollar and surroundings $\left(-26.9485^{\circ},-65.7154^{\circ}\right.$, F.E. Fernández, 01/01/1980, 1 ex. FML01259, R. Porcel, 02/14/1982, 1 ex. FML01329, O. Pagaburo, 12/10/1983, 1 ex. FML01505, M. Fernández, 01/25/1981, 1 ex. FML01512, A. Nieto. 01/10/1988, 1 ex. FML01850, O. Pagaburo, 04/24/1989, 1 ex. FML01976, G. Scrocchi, J.C. Moreta, 03/21/1992, 1 ex. FML02264, E.J. Uro, 02/11/1993, 1 ex. FML02358, P. Blazquez, 02/11/2005, 1 ex. FML16178, A. Porcel,
01/15/2015, 1 ex. FML28251). 60. Tafí del Valle and surroundings $\left(-26.8548^{\circ},-65.7116^{\circ}, \quad\right.$ C. Canzonieri, 01/01/1987, 2 ex. FML 01669, without collector information, 01/08/1983, 1 ex. FML01740, M. Corbalán, 03/01/1991, 1 ex. FML02124, F. Pape, 04/20/1993, 1 ex. FML02390, E. González, 03/11/1995, 1 ex. FML02606, J.C. Moreta, 12/04/1995, 1 ex. FML02681, without collector information, 03/06-07/1999, 1 ex. FML08274, M. Corbalán, F. Corbalán, 02/27/2000, 1 ex. FML09153, without collector information, 02/06/2001, 1 ex. FML09869, P. Caplonch, 02/15/2005, 1 ex. FML16138, G. Gallardo, 10/13/2005, 1 ex. FML16563, G. Gallardo, 12/07/2005, 1 ex. FML16642, P. Viejobueno, 03/31/2010, 1 ex. FML23633, P. Viejobueno, 04/19/2014, 1 ex. FML27182, S. Ferjancic, 04/20/2014, 1 ex. FML27185, R. Araoz, 11/01/2015, 1 ex. FML28966, J.P. Sosa, 04/01/2016, 1 ex. FML29226, without collector information, 04/20/2016, 1 ex. each, FML29318, and FML 29449, without collector, 05/20/2016, 1 ex. FML29319; J.M. Gallardo, 11/1960, 1 ex., MACN13773). 61. Km 98 ruta provincial $307\left(-26.6410^{\circ},-65.8207^{\circ}\right.$, L. Aún, R. Martori, 03/05/1993, 1 ex. FML02737). 62. Km 86 ruta provincial $307\left(-26.7161^{\circ},-65.7936^{\circ}\right.$, G. Scrocchi, M. Harvey, 01/25/2000, 1 ex. FML08973). 63. $21.7 \mathrm{~km}$ al E de Amaicha del Valle, por ruta provincial $307\left(-26.6809^{\circ}\right.$, $-65.8127^{\circ}, 2800 \mathrm{~m}$ a.s.1., L. Avila et al., 12/12/2001, 1 ex. FML13566). 64. El Pinar, Tafí del Valle $\left(-26.8145^{\circ}\right.$, $-65.7197^{\circ}$, without collector information, 03/31/2005, 1 ex. FML16380). 65. Cerca de Puesto Carpanchay, Cerro El Pichao, Sierra de Quilmes $\left(-26.3812^{\circ},-66.0810^{\circ}\right.$, 3800 m a.s.1., C. Abdala, S. Quinteros, 11/21/2006, 1 ex. FML18064). Departamento Tafí Viejo: 66. Mala Mala $\left(-26.7666^{\circ},-65.5333^{\circ}\right.$, M.G. Puchulu, 08/20/2011, 1 ex. FML24346). Departamento Trancas: 67. Quebrada de las Campanas $\left(-26.3171^{\circ},-65.6192^{\circ}, 2100 \mathrm{~m}\right.$ a.s.1., O. Pagaburp, E. Terán, 09/25/1980, 1 ex. FML01225). 68. $20 \mathrm{~km}$ de Hualinchay $\left(-26.3662^{\circ},-65.6612^{\circ}, 3100 \mathrm{~m}\right.$ a.s.1., G. Scrocchi, S. Quinteros, 12/06/2004, 1 ex. FML16131). 69. $12.2 \mathrm{~km}$ desde curva para subir desde Hualinchay $\left(-26.3280^{\circ},-65.6209^{\circ}, 2500 \mathrm{~m}\right.$ a.s.l., F. Lobo et al., 12/15/2001, 1 ex. MCN-UNSa733).

BOLIVIA. La Paz, (without collector information, 1919, 1 ex. FML00464). CHILE. Pro. Entre San Bernardo de las Zorras y El Rosal, Ruta Provincial 127, $21.3 \mathrm{~km}$ NE de Ruta Nacional $51\left(-24.4025^{\circ},-65.7768^{\circ}, 3300\right.$ $\mathrm{m}$ a.s.1., T. Hibbard et al., 04/07/2017, 1 ej. IBIGEOR5717-5718). Provincia de Atacama: Afueras del Parque Nacional Llanos de Challe $\left(-28.1523^{\circ},-71.0297\right.$, without collector or date information, 1 ex. MACN40056). PERÚ. Huamahuaco, (W. Weyrauch, without date information, 1 ex. FML01132). Departamento Puno: Sillustani, (E. Lavilla, 10/06/1983, 1 ex. FML 01491).

\section{Discussion}

The locality El Parrón, where the specimen FML30163 was found, is approximately $190 \mathrm{~km}$ SW from the nearest previously known locality (Cerro El Manchao, 
Catamarca, marked in the map in Scrocchi et al. 2006). Also, El Parrón and Cueva de Logroño in Famatina area are the southernmost records of the distribution of Tachymenis peruviana. The highlands in NW Argentina, especially the regions of highest altitude, which include most of the distributional area of Tachymenis peruviana in the country, have problems regarding protection and conservation, such as soil erosion, mining, sports with off-road vehicles, infrastructure works such as gas pipelines and high-voltage lines (González 2005). Therefore, although it is considered a non-threatened species in the country (Giraudo et al. 2012b), it is important to analyze periodically the situation of its populations.

We also revised other type material of Tachymenis peruviana yutoensis, considered junior synonym of Tachymenis peruviana (Terán 1988, Wallach et al. 2014), in addition to those mentioned in "Other material..." section. The holotype (CENAI2632) is labeled "Yuto, Departamento Ledesma, Jujuy" ( $\left.-23.6469^{\circ},-64.4732^{\circ}\right)$, a locality of low plains (350-400 m a.s.1.), with forests and warm climate, which according to the known ecological requirements for $T$. peruviana, would be unsuitable for the species. The paratypes (CENAI2633-2634) come from "Santa Bárbara", which is a mountain chain in Jujuy, where T. peruviana does occur (Fig. 3), but without more specific locality data in the collection record, which does not allow to locate the exact collection site in the map.

\section{Acknowledgements}

We are grateful to Emanuel Luna Toledo for the photographs of the specimen from Cueva de Logroño, to Demián Slodki who helped us with the collection data of MCN and IBIGEO; and to Pablo Pereyra for drawing the maps. Also, we would like to thank J. Faivovich (MACN), E. Lavilla and S. Kretzschmar (FML), for the permissions to revise the collections at their care.

\section{Authors' Contributions}

GAG, SJN and GJS collected the data, wrote, and reviewed the text.

\section{References}

Aparicio J (1994) Herpetofauna: Anfibios y Reptiles. In: de Morales CB (Ed) Huaraco, Comunidad de la Puna. Instituto de EcologíaUniversidad Mayor de San Andrés, La Paz, 115-125.

Cabrera MP, Stazzonelli JC, Scrocchi GJ (2017) Sapos, ranas, lagarti- jas y serpientes de los Valles Calchaquíes (Catamarca, Tucumán y Salta, Argentina). Serie Conservación de la Naturaleza 23. Fundación Miguel Lillo, Tucumán, Argentina, 108 pp.

Cabrera AL, Willink A (1980) Biogeografía de América Latina. OEA, Serie Biología, Monografía 13: 1-122.

Cei JM (1993) Reptiles del noroeste, nordeste y este de la Argentina. Bolletino Museo Regionale di Scienze Naturali, Torino, Monografie 4: 1-949.

Demangel D (2016) Reptiles en Chile. Fauna Nativa Ediciones, Santiago, Chile, $619 \mathrm{pp}$.

Giraudo A, Scrocchi GJ (2002) Argentinean snakes: an annotated checklist. Smithsonian Herpetological Information Service 132: $1-53$

Giraudo AR, Vidoz F, Arzamendia V, Nenda SJ (2012a) Distribution and natural history notes on Tachymenis chilensis chilensis (Schlegel, 1837) (Reptilia: Serpentes: Dipsadidae) in Argentina. Check List 8 (5): 919-923. http://doi.org/10.15560/8.5.919

Giraudo A, Arzamendia V, Bellini GP, Bessa CA, Calamante CC, Cardozo G, Chiaraviglio M, Costanzo MB, Etchepare E, Di Cola V, Di Pietro D, Kretzschmar S, Palomas S, Nenda SJ, Rivera P, Rodríguez ME, Scrocchi GJ, Williams JD (2012b) Categorización del estado de conservación de las Serpientes de la República Argentina. Cuadernos de Herpetologia 26 (Supl. 1): 303-326.

González JA (2005) Los ambientes naturales en áreas montañosas del Noroeste Argentino (NOA), su interrelación con países limítrofes y su necesidad de protección, recuperación y conservación. Serie Conservación de la Naturaleza 15. Fundación Miguel Lillo, Tucumán, Argentina, 28 pp.

IUCN (2018) Red List of Threatened Species. Version 2017-3. http:// www.iucnredlist.org. Accessed on: 2018-05-22.

Nenda SJ, Blotto BL, Pereyra MO, Pinheiro PDP, Giraudo A (2017) Occurrence of Tachymenis chilensis chilensis (Schlegel, 1837) (Reptilia: Serpentes: Dipsadidae) and other herpetological fauna from Epu Lauquen Natural Protected Area, Neuquén, Argentina. Check List 13 (2): 2079. http://doi.org/10.15560/13.2.2079

Ramos VA (1999) Las provincias geológicas del territorio argentino. Geología Argentina 29 (3): 41-96.

Scrocchi GJ, Moreta JC, Kretzschmar S (2006) Serpientes del Noroeste Argentino. Fundación Miguel Lillo, Tucumán, Argentina, 178 pp.

Scrocchi G, Abdala C, Nori J, Zaher H (2010) Reptiles de la provincia de Río Negro, Argentina. Fondo Editorial Rionegrino, Viedma, Río Negro, Argentina, $252 \mathrm{pp}$.

Szumik C, Molina A, Rajmil J, Aagesen L, Correa C, Pereyra VV, Scrocchi GJ (2016) El maravilloso mundo de los animales y plantas de la Puna. Alfarcito y Laguna de Guayatayoc, Jujuy, Argentina. Serie Conservación de la Naturaleza 22. Fundación Miguel Lillo, Tucumán, Argentina, 173 pp.

Terán EMT (1988) Estudios sobre el status taxonómico y distribución ecogeográfica de Tachymenis peruviana Wiegmann (Serpentes: Colubridae) en el noroeste argentino. Boletín Asociación Herpetológica Argentina 4 (2-3): 14.

Wallach V, Williams KM, Boundy J (2014) Snakes of the World. A Catalogue of the Living and Extinct Species. Taylor \& Francis Group, Boca Raton, Florida, USA. 1227 pp.

Williams JD, Scrocchi GJ (1994) Ofidios de agua dulce de la República Argentina. In: Castellanos ZA (Ed) Fauna de agua dulce de la República Argentina 42: 1-55. 